\title{
Étude et développement d'une source de pompage optique VUV de forte puissance pour l'amplification d'impulsions laser femtosecondes sur la transition $\operatorname{XeF}(\mathrm{C}-\mathrm{A})$
}

\author{
V.I. Tcheremiskine, M. Sentis, L.D. Mikheev ${ }^{1}$ et R. Clady \\ Laboratoire Lasers, Plasmas et Procédés Photoniques, LP3, FRE 2165 du CNRS, \\ Université d'Aix-Marseille II, Campus de Luminy, Case 917, 13288 Marseille cedex 9, France \\ ${ }^{1}$ Laboratoire des Processus Photochimiques, Institut de Physique P.N. Lebedev, \\ Leninsky Prospekt 53, Moscou 119991, Russie
}

\begin{abstract}
Résumé : Le rayonnement UV-VUV issu d'un plasma généré par une décharge électrique de forte puissance dans un milieu gazeux (ici $\mathrm{XeF}_{2}$ ) est capable d'exciter de grands volumes de différents milieux lasers. Nous présentons une nouvelle source optique UV-VUV de forte puissance basée sur une décharge multi-canaux réalisée sur la surface d'un diélectrique ainsi que les premiers résultats d'amplification d'impulsions femtosecondes obtenus dans le milieu actif d'un laser photolytique $\mathrm{XeF}(\mathrm{C}-\mathrm{A})$. Ce milieu est très attractif pour développer des systèmes lasers hybrides de forte puissance (petawatt) dans le domaine du visible. En effet, la transition $\mathrm{XeF}(\mathrm{C} \sim \mathrm{A})$ est caractérisée par une bande spectrale très large dans le bleu-vert $(460-520 \mathrm{~nm})$ permettant d'amplifier des impulsions optiques d'une durée de l'ordre de 10 fs.
\end{abstract}

\section{INTRODUCTION}

Les systèmes laser femtoseconde de forte puissance (domaine Terawatt voire Petawatt) constitués d'éléments actifs solides (Titane - Saphir, Nd:verre) sont soumis à des limitations de puissance et de qualité de faisceau liées aux caractéristiques des matériaux utilisés (faible conductivité thermique, importance des nonlinéarités, dimensions réduites). Une solution alternative aux systèmes lasers "tout solide" est l'utilisation d'un milieu gazeux comme étage final d'amplification. Les avantages inhérents à l'utilisation d'un milieu actif gazeux, sont :

- un seuil d'apparition des phénomènes non linéaires beaucoup plus élevé ( 3 ordres de grandeur typiquement). Ainsi, la faible non - linéarité du milieu gazeux permet théoriquement l'amplification directe d'impulsions ultrabrèves de forte énergie (jusqu'à $10 \mathrm{~J}$ ) sanș l'emploi de systèmes d'étirement et de recompression.

- la possibilité d'utiliser de très grandes sections et longueurs de milieu actif permettant un dimensionnement aisé et donc d'atteindre des énergies très élevées.

Parmi les milieux gazeux possibles, le laser XeF (C-A) présente des caractéristiques exceptionnelles d'amplification : une largeur de gain importante $(80 \mathrm{~nm}$ centrée à $474 \mathrm{~nm})$, une énergie de saturation élevée $(0,05$ $\left.\mathrm{J} / \mathrm{cm}^{2}\right)$ et une faible section d'émission stimulée $\left(10^{17} \mathrm{~cm}^{2}\right)$. De telles propriétés permettent, théoriquement, l'amplification d'impulsions ultrabrèves $(10 \mathrm{fs})$ et d'atteindre une puissance crête de $40 \mathrm{TW} / \mathrm{cm}^{2}\left(\mathrm{P}_{\mathrm{th}}=h v \Delta v / \sigma\right)$. Ces chiffres sont tout à fait comparables à ceux du Nd-glass $\left(80 \mathrm{fs}\right.$ et $\left.60 \mathrm{TW} / \mathrm{cm}^{2}\right)$ et du Titane - Saphir $(8 \mathrm{fs}$ et $120 \mathrm{TW}$ $\mathrm{cm}^{2}$ ).

De plus, outre les avantages précédemment évoqués (quasi - absence d'effets non - linéaires permettant la suppression des étages d'étirement et de compression, grande section d'amplification et grande longueur de gain), le milieu XeF présente l'intérêt d'émettre dans le domaine spectral du bleu vert. Une très forte luminance liée à la courte longueur d'onde pourra ainsi être atteinte avec une puissance laser significativement plus faible par rapport aux milieux amplificateurs solides (émission dans l'infrarouge) dans le cas du laser XeF (C-A). Cette solution hybride (laser solide / amplificateur à gaz) a, de plus, l'avantage d'améliorer grandement le contraste qui est un problème majeur pour l'utilisation des lasers ultrabrefs de puissance. Lorsque de tels lasers sont focalisés sur une cible, il est en effet primordial de limiter l'influence des préimpulsions. Dans le présent schéma, l'impulsion utile, avant amplification, est obtenue par effet non - linéaire et comme l'efficacité de cette conversion est beaucoup plus importante pour l'impulsion que pour ses pieds (pré et post - impulsions), elle acquiert ainsi un contraste initial avant amplification très élevée. Par la suite, les propriétés du milieu XeF (optimisation du produit gain x longueur de gain) et la géométrie du schéma d'amplification doivent permettre de conserver un contraste élevé

Le laboratoire LP3 étudie donc un laser femtoseconde hybride émettant dans le domaine du visible à partir d'un oscillateur solide (Titane - Saphir) amplifié dans un milieu gazeux (laser photochimique XeF (C-A)) après conversion par effet non - linéaire (OPA). Dans le futur, l'amplificateur sera directement injecté par le $2^{\text {ínu }}$ harmonique d'un dispositif Ti:Sa fonctionnant à la longueur d'onde de $950 \mathrm{~nm}$. 


\section{SOURCE DE POMPAGE}

La méthode de pompage de l'étage amplificateur est basée sur la photodissociation de la molécule $\mathrm{XeF}_{2}$ par un rayonnement VUV $(160 \mathrm{~nm})$ conduisant à la formation de XeF dans l'état $\mathrm{B}$ qui ensuite se relaxe par effet collisionel dans l'état C. Une source optique VUV impulsionnelle originale a été réalisée ; elle présente une surface radiative de grandes dimensions $\left(40 \times 17 \mathrm{~cm}^{2}\right)$ avec une durée d'impulsion optique inférieure à $1 \mu$ s. La décharge est composée de 50 canaux placés en parallèle $(17 \mathrm{~cm}$ de long, séparés de $8 \mathrm{~mm})$ initiés simultanément dans un mélange gazeux (principalement azote et gaz rare) sur la surface d'une plaque en Téflon. La figure 1 est une représentation schématique du circuit de décharge.

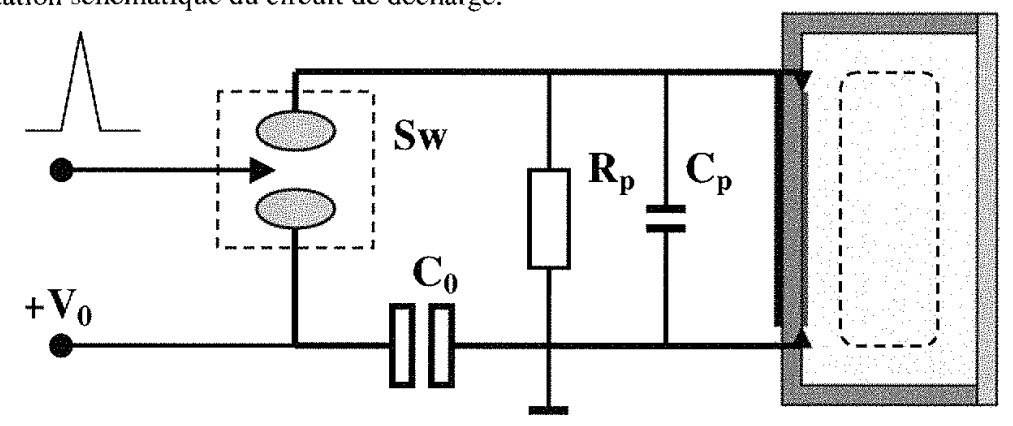

Figure 1 : Représentation schématique du circuit de décharge.

La décharge est alimentée par 5 condensateurs $\left(\mathrm{C}_{0}\right)$ de $0,6 \mu \mathrm{F}$, qui peuvent être chargés jusqu'à une tension $\left(\mathrm{V}_{0}\right)$ de $45 \mathrm{kV}$. Le circuit électrique de décharge est alors commuté à l'aide d'un éclateur multi-canaux (Sw) de faible inductance conçu spécialement. La température de brillance du plasma radiatif dans le domaine spectral VUV a été mesurée par une méthode d'actinométrie dynamique [1]. Les mesures montrent que cette température excède $20000 \mathrm{~K}$ au maximum du courant de la décharge $\left(I_{\max }=160 \mathrm{kA}\right)$.

L'étude du transport d'un rayonnement incohérent large bande dans un milieu absorbant et photodissociatif (molécules $\mathrm{XeF}_{2}$ ) a été réalisée afin d'obtenir un régime homogène đ'amplification d'impulsions femtosecondes. Pour atteindre une telle caractéristique, il est important d'avoir une meilleure connaissance des processus de photodissociation induits par la source de pompage. Pour cela, une nouvelle approche analytique a été développée permettant la description des processus de formation de l'onde de photodissociation [2]. L'étude théorique permet de démontrer que pour n'importe quel milieu absorbant, la dynamique de l'onde de photodissociation peut être décrite par une approche asymptotique tendant toujours vers la solution stationnaire. Elle permet également de calculer la densité optimale du milieu absorbant $\left(\mathrm{XeF}_{2}\right)$ pour obtenir une amplification homogène d'un faisceau sonde de grande dimension transversale $(3$ à $6 \mathrm{~cm}$ ). A titre d'exemple, la figure 2 montre qu' avec un pompage du milieu actif à l'aide de deux sources placées en parallèle, il est possible pour une température de brillance de la source donnée $(24 \mathrm{kK})$ d'obtenir un gain homogène sur une largeur de $3 \mathrm{~cm}$ si la concentration de $\mathrm{XeF}_{2}$ est de 2 . $10^{16} \mathrm{~cm}^{-3}$.

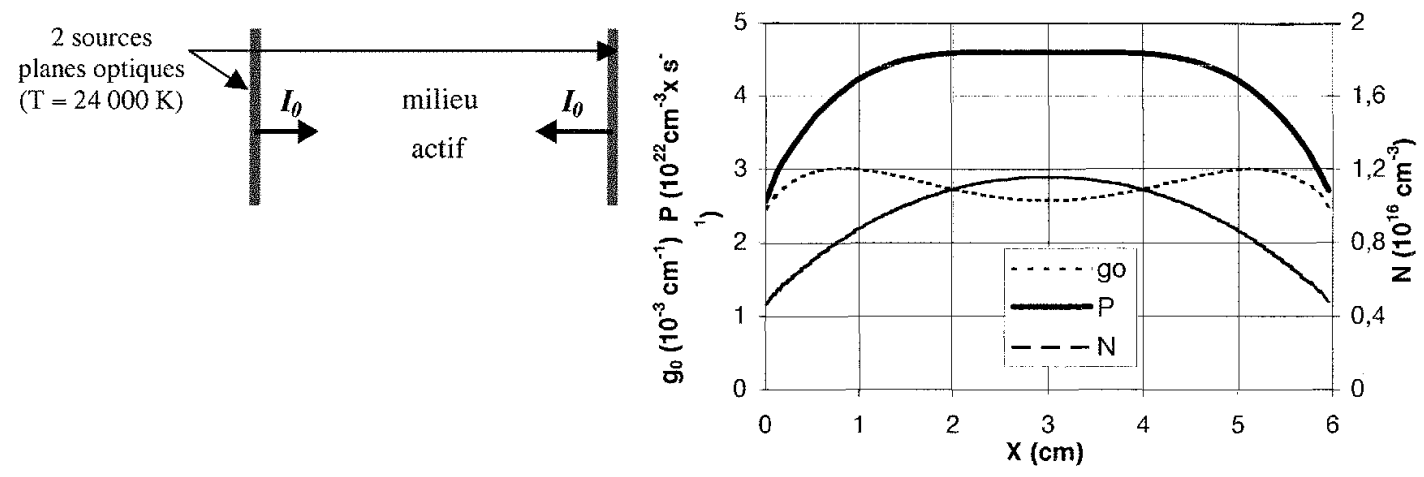

Figure 2: Gain à petit signal $g_{0}(\ldots .$.$) , taux de pompage dans l'état \mathrm{XeF}(\mathrm{B})\left(\mathrm{P} \quad\right.$ _ et concentration de $\mathrm{XeF}_{2}(\mathrm{~N}$---) pour deux sources optiques de pompage placées en parallèle délivrant $5.10^{23}$ photons VUV/cm ${ }^{2} \mathrm{~s}\left(\mathrm{I}_{0}\right)$ et un mélange composé de $\mathrm{XeF}_{2} / \mathrm{N}_{2} / \mathrm{Kr}: 2.10^{16} / 1,2.10^{18} / 2,4.10^{19} \mathrm{~cm}^{-3}$. 


\section{ETUDE DE L'AMPLIFICATION}

Une étude paramétrique du gain résolue dans le temps et dans l'espace en fonction de la composition du mélange gazeux et de l'intensité de rayonnement d'une source de photodissociation a tout d'abord été entreprise. Cette étude a été réalisée à l'aide d'un laser argon continu à la longueur d'onde de $488 \mathrm{~nm}$, proche du maximum de la bande d'amplification de la transition XeF (C-A). Une amplification égale à 10 a été mesurée dans la configuration optimale pour une concentration de $\left[\mathrm{XeF}_{2}\right]$ égale à $410^{16}$ atomes $/ \mathrm{cm}^{3}$.

Ensuite l'assemblage d'un système hybride (voir figure 3) composé d'un laser Ti:Sa, d'un OPA et de l'amplificateur XeF a été réalisé. Le laser Ti:Sa associé à l'OPA permet d'obtenir une impulsion d'une durée de -130 fs et de qq $\mu \mathrm{J}$ accordable dans le domaine du bleu vert $(460-520 \mathrm{~nm})$.

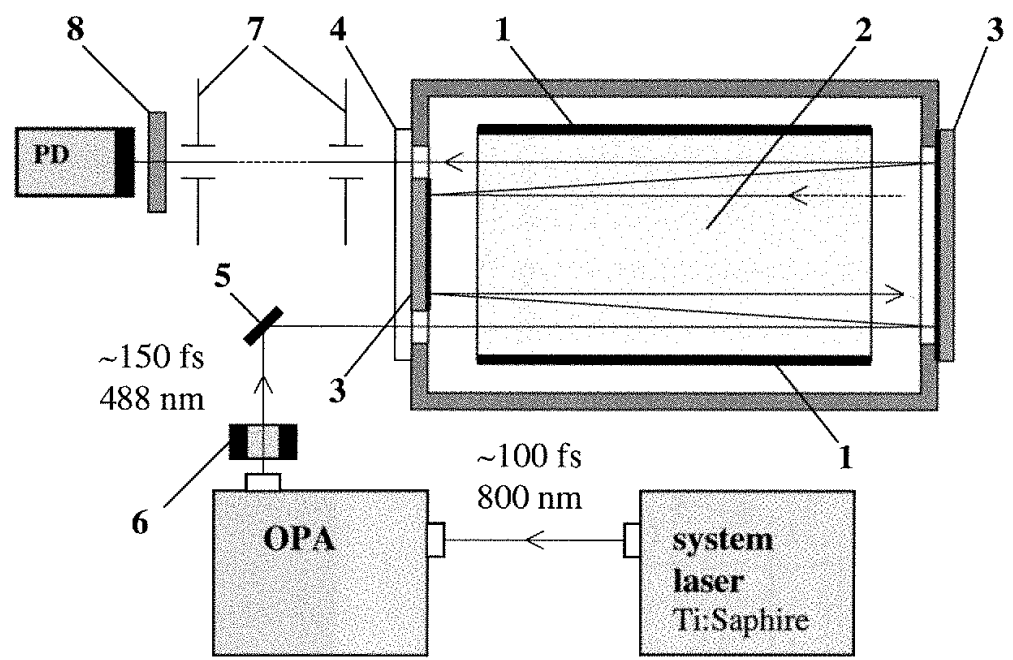

Figure 3 : Représentation schématique du laser hybride : 1 Plasma de décharge, 2 volume de décharge, 3 miroir dichroïque, 4 fenêtre $\mathrm{CaF}_{2}, 5$ miroir large bande, 6 obturateur, 7 diaphragmes, 8 filtre bande étroite.

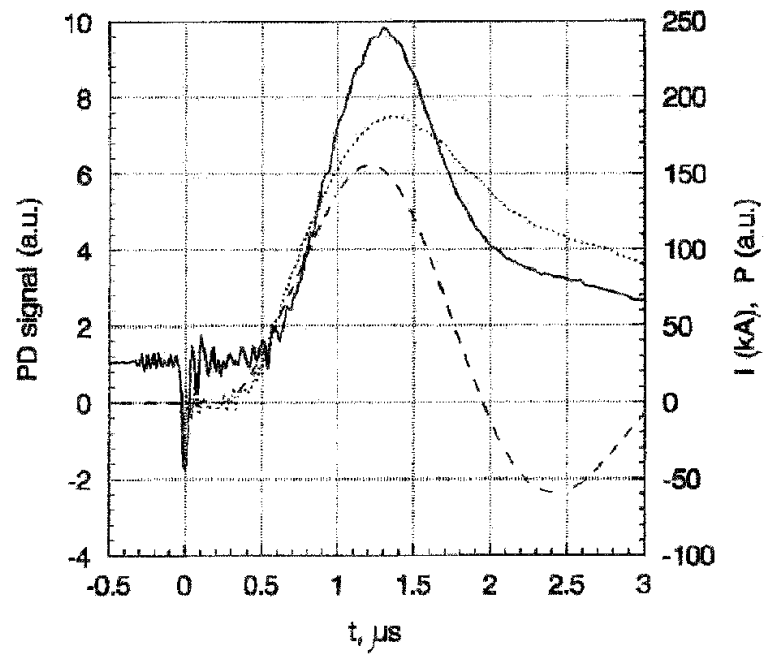

Figure 4 : Evolution temporelle du courant de décharge (I ....), de l'intensité lumineuse du plasma de décharge (P ........) et du signal de photodiode ( 
La figure 4 correspond à la mesure du gain pour une impulsion femtoseconde de $488 \mathrm{~nm}$. Cette figure montre l'évolution temporelle du courant de décharge (I), de l'intensité lumineuse du plasma de décharge (P) et du signal de photodiode. Lorsque l'impulsion provenant de l'OPA est injectée au moment ou le gain du milieu amplificateur atteint son maximum le gain total mesuré est de 5 ce qui correspond à gain à petit signal de $1,610^{-3} \mathrm{~cm}^{-1}[3]$.

\section{CONCLUSION}

Cette étude préliminaire a montré pour la première fois qu'un système hybride femtoseconde avec comme étage final d'amplification un laser photodissociatif était une solution originale et prometteuse pour l'obtention d'impulsions laser femtosecondes de très forte énergie dans le domaine du bleu vert avec un très bon contraste. L'adjonction dans le futur d'une deuxième source de pompage doit permettre en augmentant la tension de charge et en utilisant du krypton comme gaz tampon d'obtenir un gain à petit signal de $510^{-3} \mathrm{~cm}^{-1}$ correspondant à un gain total de 100 pour une longueur d'amplification de $10 \mathrm{~m}$. Ces valeurs permettraient en injectant une impulsion de 30 fs et $1 \mathrm{~mJ}$ d'obtenir en sortie d'amplificateur une impulsion de $0,1 \mathrm{~J}$ sans utiliser un système d'étirement et de recompression pour un faisceau ayant une section de $10 \mathrm{~cm}^{2}$.

\section{Remerciements}

Ce programme a reçu le soutien de la Région PACA, du Conseil Général 13, de la Ville de Marseille ainsi que de l'Europe à travers le programme SHARP (Contrat HPRI-CT-2001-50037).

\section{Références}

[1]. V.I. Tcheremiskine, M.L. Sentis, L.D. Mikheev, et al., J. Appl. Phys. 80 (4), 2094-2096, (1996).

[2]. V.I. Tcheremiskine., L.D. Mikheev, M.L. Sentis, V.S Zuev. Photodecomposition waves driven by a broadband arbitrarily directed radiation. Physical Review A, 36, p 13808, (2001)

[3]. V.I. Tcheremiskine, M.L. Sentis, L.D. Mikheev. Amplification of ultrashort laser pulses in the photolytically driven XeF (C-A) active medium. Applied Physics Letters, 81, p 403, (2002). 